\title{
The long term outcome in 149 patients with spinal cord injury
}

\author{
P Daverat $^{1}, \mathrm{H}$ Petit $^{2}, \mathrm{G} \mathrm{Kemoun}^{2}$, JF Dartigues ${ }^{3}$ and M Barat ${ }^{2}$ \\ ${ }^{1}$ Department of Medical Rehabilitation, Hôpital Saint-André, 33075 Bordeaux Cédex; ${ }^{2}$ Department of Medical \\ Rehabilitation, Hopital Pellegrin, Bordeaux; ${ }^{3}$ INSERM U330 Université de Bordeaux II, Bordeaux, France
}

\begin{abstract}
A longitudinal follow-up study of 149 spinal cord injured patients is presented. Ninety two patients have been visited and interviewed at home 7-10 years after injury (28\% died during the follow-up). Disability was assessed using the Functional Independence Measure (FIM), and was correlated with the neurological level, impairment and spasticity. The handicap was assessed using the Reintegration to Normal Living Index (RNLI). Multivariate analysis showed a correlation with age, neurological impairment, sexual impairment, living conditions and social life. There was a high correlation with depression and the Functional Independence Measure. The early neurological examination correlated with their long term disability but not with their handicap.
\end{abstract}

Keywords: spinal cord injury; disability; handicap; outcome

\section{Introduction}

Spinal cord injury (SCI) occurs suddenly, without warning, and causes dramatic changes in individuals' lives. The broadness of the physiological changes accompanying SCI underscores the importance in continuing to improve rehabilitation programmes, so as to assist patients to regain control of their lives after injury.

The study of the mechanisms that produce handicaps requires three dimensions: impairment, disability, and socioeconomic factors. The long term outcome of patients with SCI has been already studied in many ways and there is certain agreement concerning impairment and disability. ${ }^{1,2}$

There are still many differences seen in the assessment of social and professional reintegration, which depend on the social and cultural systems of each country. ${ }^{2-8}$ In France, data concerning outcome remain poor. One retrospective study with $45 \mathrm{SCI}$ patients, 1-8 years after injury, has been published already. ${ }^{9}$

This study presents a longitudinal prospective analysis of 188 patients with SCI admitted after injury directly to the emergency room of the Pellegrin Hospital (Bordeaux, France). The aims are to:

- describe the handicap of a representative cohort of SCI patients in Aquitaine (South-West region of France)

- analyse the correlation between impairment, and disability and the environmental factors.

\section{Patients and methods}

Procedure

Data were collected from January 1982 to December 1985 , on a consecutive sample of 188 SCI patients initially admitted to the emergency ward of the
Pellegrin Hospital (Bordeaux, France). The initial neurological status was assessed within $6 \mathrm{~h}$ after admission. Each patient was followed up regularly during the first year. ${ }^{10}$ Thirty-nine patients $(21 \%)$ died during this period.

In 1992, the 149 surviving patients were introduced to the study by telephone or letter, and were invited to participate in the study. We were told that 14 more had died. Among the 135 survivors, 27 were lost to follow-up and could not be reached at their initial address. Sixteen patients refused to participate. The reason for non-participation was not known in four cases; a lack of interest in five cases; moving out of the area in four cases; and a negative attitude towards the hospital in three cases.

The ninty-two patients studied $(68 \%$ of survival subjects) were visited at home by a physician and/or an occupational therapist and are reported here.

\section{Instruments}

The assessment questionnaire was constructed in three parts, and included impairment, disability, and socioeconomic factors.

The Yale Scale Score (YSS) ${ }^{11}$ is a quantitative measurement that allows grading of the lesional and sublesional neurological findings. The score is determined by motor and sensory testing, each on a scale of five points. The total score varies from 0 to 10: 0 indicates a complete lesion, while 10 corresponds to intact medullary function.

The depressive symptomatology was assessed by the Centre for Epidemiological Studies Depression Scale (CES-D). ${ }^{12}$ The CES-D is a short (20 items), selfreport measure that takes approximately $15 \mathrm{~min}$ to complete. Respondents are asked how often over the 
past week they have experienced each of 20 symptoms, rated on a four-point scale. The total sum of the responses ranges from 0 to 60 , the higher scores indicating more symptoms of depression, weighted by frequency of occurrence during the past week. The cut-off in France was 17 in men, and 23 in women. It indicated probable morbidity.

Patients were also interviewed on other items, without quantification: incontinence, sexual life, skin, pain, and orthopaedic status.

Disability was assessed using the Functional Independent Measure (FIM) ${ }^{13}$ that includes 18 items, rated on a seven-point scale. The total sum of the responses ranges from 18 to 126 , with the highest score indicating the greatest maximum independence in daily living activities.

To measure the various dimensions of handicap, participants used the Reintegration to Normal Living Index (RNLI). ${ }^{14}$ The RNLI is a self-perceived questionnaire that appears to assess global function. It measures both the patients' perceptions of their own capabilities and the objective indicators of physical, social and psychological performance. The RNLI evaluates eight areas related to activity and daily living: mobility indoor; mobility in the community and out-oftown; self care skills; daily and working activities; recreational activities; and social and family role measures (daily function subscales). The RNLI also measures three areas related to self perception: personal relationships, self presentation, and general coping skills. Thus a total of 11 questions were asked, rated on 10-point analogic visual scale. The total sum is ranged on a 100-point scale. Previous research has demonstrated validity of the French version in Quebec. ${ }^{14}$

Socioeconomic factors were also determined: family status, social relationships, education, mobility within one's home and community, health care needs.

\section{Statistical analysis}

Univariate analysis was first performed using the $\chi^{2}$ test, Student's $t$ test and the Spearman test according to the type of variable. Multilinear regression analysis was used to correlate the FIM and the RNLI to other variables. The MIF was tested with a combination of age, sex, CES-D, Yale Scale Score, and the following factors. Spasticity, orthopaedic limitations, pain, and urinary incontinence were assessed as present (yes) or absent (no), (yes or no). The RNLI was tested with a combination of age (by year), Yale Scale Score, FIM, CES-D, relationships (decreased or not), living conditions (alone or not), sexual life (yes or no), moving freely in living quarters (yes or no), education level (complete secondary or not), current work status (employed or not), and leisure (yes or no).

\section{Results}

The total initial sample consisted of 188 patients with SCI. Within this population, $151(80.3 \%)$ were male, ranging in age from 5 to 84 years (mean age: 38 years), with 97 tetraplegic individuals (51.6\%). Upon admission to hospital, $95(50.5 \%)$ had a complete lesion $(\mathrm{YSS}=0)$. The mean YSS was 2.

In 1992, 92 patients were visited at home. Within this population, $73(79.4 \%)$ were male, with a ratio of male:female 3.8 , ranging in age from 13 to 83 years (mean age: 39 years). Forty three were tetraplegic $(46.7 \%)$; among those who were tetraplegic, 12 $(28.6 \%)$ had a complete lesion (YSS $=0$ ). Among those who were paraplegic, $24(48 \%)$ had a complete lesion.

Urinary incontinence was present in 52 patients $(57 \%)$, spasticity in $41(45 \%)$. Twenty-eight $(64 \%)$ of the tetraplegic patients and $17(46 \%)$ of those who were paraplegic experienced pain daily. Depression was noted in 33 patients $(36.3 \%)$, and was not correlated with the level of the lesion (whether paraplegic or tetraplegic), nor with the YSS.

The FIM scores according to the level of the lesion are shown in Figure 1. The mean score for the total sample was $99.4 \pm 25$. It was $109.7 \pm 16.2$ for paraplegic patients, and $87.1 \pm 28$ for those who were tetraplegic. This clearly indicates that most of those who were paraplegic living at home are as would be expected, independent in activities of daily living.

There appears to be a strong correlation between the initial YSS and the FIM $(P<0.001)$. The multivariate analysis shows that the FIM is correlated with the YSS $(P<0.001)$, spasticity $(P<0.002)$ and pain $(P=0.02)$. The YSS and spasticity contributed to $35 \%$ of the FIM variance. The FIM was not correlated with age, sex, urinary incontinence and orthopaedic limitations.

The RNLI scores according to the level of the lesion are shown in Figure 2. The mean score for the total sample was $78.4 \pm 17.7$. It was $81.8 \pm 14.5$ for those who were paraplegic, and $74.4 \pm 20.3$ for those who were tetraplegic. There was no statistical difference between these two populations. Figure 3 shows the RNLI scores for each item as a function of the level of the lesion. These scores are practically parallel between

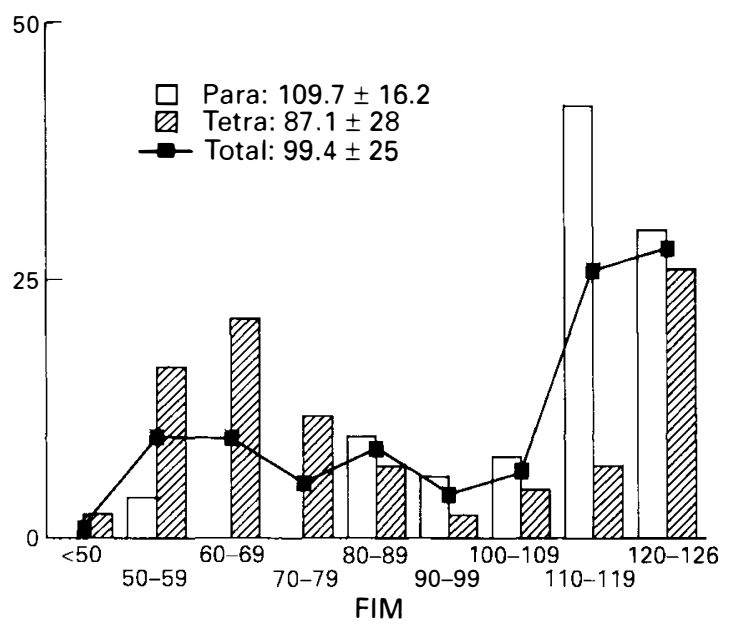

Figure 1 FIM as a function of the level of the lesion (percentage) 


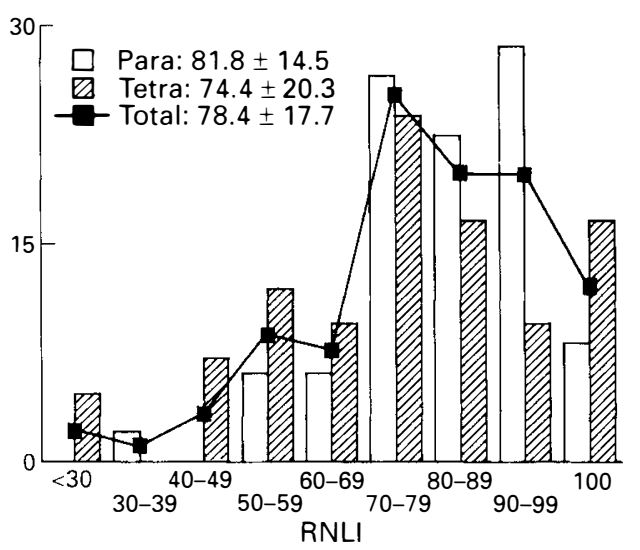

Figure 2 RNLI as a function of the level of the lesion (percentage)

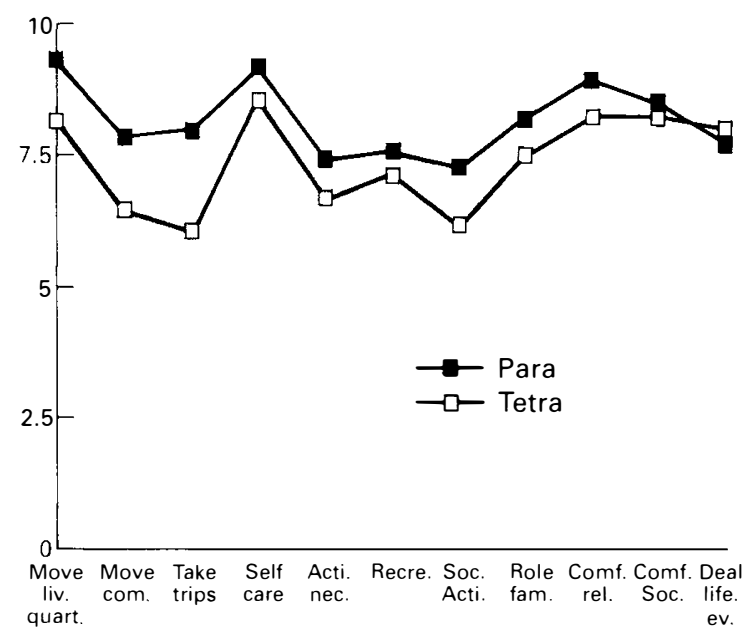

Figure 3 RNLI items scores for paraplegic and tetraplegic patients

paraplegic and tetraplegic subjects, with approximatively one point difference.

The univariate analysis shows that there is no correlation between the RNLI and the initial YSS, but the RNLI is significantly correlated with the FIM $(P<0.001)$. The multivariate analysis shows seven significant variables for RNLI, as shown in Table 1: age $(P=0.01)$, YSS $(P=0.04)$, living conditions $(P=0.01)$, relationships $(P=0.01)$, CES-D
$(P<0.001)$, FIM $\quad(P<0.001)$, and sexual life $(P=0.05)$. These seven independent variables contributed to $72 \%$ of the RNLI variance. The RNLI items mostly correlated with the FIM are mobility in living quarters and in the community. The RNLI items mostly correlated with depression are participating in social activities, role in family, and dealing with life events.

\section{Discussion}

Some limits of this study have to be underlined. Only 92 patients ( $68 \%$ of survival subjects) have been visited at home: 27 were lost to follow-up and 16 refused to participate. Among the patients that were included, there were no missing data. All of them were tested with the RNLI method.

The Yale Scale Score was chosen in 1981 just before the beginning of the study. It was at this time the most pertinent instrument to evaluate neurological impairment. The ASIA and after the ASIA-IMSOP score was not used as it is today.

Long term independence in SCI patients is clearly related in our study to the lesional level and the severity of the lesion (YSS). The mean FIM scores are comparable with other major studies, either at discharge, ${ }^{1}$ or in the long term. ${ }^{2}$ This tends to show a stability in disabilities during the first 10 years.

The strong correlation between the initial neurological examination (YSS) and the long term autonomy shows the limits of rehabilitation, dealing with a stable impairment. On the contrary, there was no correlation between the initial YSS and RNLI. Many factors determine handicap: age, neurological impairment, depression, sexual life, disabilities, and social integration (living conditions, relationships). These are extrinsic factors common to all multiple injuries, and not specifically related to a spinal cord lesion. Another explanation could be that several years passed between the first and second measurement, so the reliability between assessors may be relevant and may explain why the initial YSS did not correlate to the RNLI.

Some predictors already described in the literature were not found in our study: urinary incontinence, pain, employment after injury, leisure. ${ }^{2,6,7,15}$ Some of them were correlated to RNLI with univariate analysis, but were not found as independent predictors with the multivariate analysis. This confusion bias has not been always analysed in other studies. Another possible

Table 1 Multilinear regression analysis of RNLI

\begin{tabular}{lccc}
\hline & Coefficient & Standard error & $P$ \\
\hline Age (by year) & -0.27 & 0.10 & 0.01 \\
Yale Scale Score (score) & -0.99 & 0.47 & 0.04 \\
FIM (score) & 0.34 & 0.06 & $<0.001$ \\
CES-D (score) & -0.87 & 0.14 & $<0.001$ \\
Living conditions (alone vs not) & 9.02 & 3.26 & 0.01 \\
Relationships (decreased vs not) & -7.39 & 2.91 & 0.01 \\
Sexual life (yes $v s$ no) & -6.96 & 3.47 & 0.05 \\
\hline
\end{tabular}

$P<0.05$ 
explanation is the low level of quantification in our study (yes or no). This may be particularly relevant for pain.

There is some controversy in the literature about the effect of age on handicap. Age was considered to be an increasing factor in some studies. ${ }^{16}$ In our study and in others, ${ }^{17}$ it was found to be a better reintegration factor.

Depression was found, in agreement with the literature, to be correlated with RNLI. ${ }^{18}$ Its prevalence in our study was high, and it was not related to neurological impairment. Its significance is difficult to analyze: it could be a cause of the difficulties to reintegrate normal living conditions, or a consequence of poor reintegration. It could also be a high preinjury disposition.

Living arrangements and relationships were found to be related to handicap in our study as was the case in many other reports. ${ }^{2,7}$ Most of the authors found no correlation between life satisfaction and neurological impairment or disability. ${ }^{2}$ In our study, reintegration was dependent on these two factors, but RNLI does not only analyse quality of life and contains many items concerning mobility.

\section{Conclusion}

From the present results, we can conclude that among the SCI patients admitted to one hospital on the same day after the accident, the long term outcome was satisfactory in terms of disability. Most of the paraplegic individuals were independent, whatever were the social or the environmental factors. The quality of the social reintegration was multifactorial. Some factors appeared to have been neglected, such as depression or chronic pain. Most of the patients lacked information about some specific treatments: sexual function, fertility, and upper limb functional surgery for tetraplegic subjects. More efforts have to be made in our country.

\section{Acknowledgements}

This work was supported by a grant from the Institut National de la Santé et de la Recherche Médicale (CRE INSERM no. 921101).

\section{References}

1 De Vivo MJ, Richards JS, Stover SL, Go BK. Spinal cord injury. Rehabilitation adds life to years. West J Med (special issue) $1991 ;$ 154: 602-606.

2 Siosteen $\mathrm{A}$ et al. The quality of life of three functional spinal cord injury subgroups in a Swedish community. Paraplegia 1990; 28: 476-488

3 Lang HD, Durr W, Hoffman J, Koeth R. Post-clinical follow-up of spinal patients through domestic checkups (Koblenz model). Paraplegia 1980; 18: 140-148.

4 Jenik F, Kuhn W, Zach GA. Social and vocational reintegration of paraplegic and tetraplegic patients in Switzerland. Paraplegia 1982; 20: 65-70.

5 Sutton RA et al. Review of the social situation of paraplegic and tetraplegic patients rehabilitated in the Hexham regional spinal injury unit in the north of England over the past four years. Paraplegia 1982; 20: 71-79.

6 Jakajima A, Honda S. Physical and social condition of rehabilitated spinal cord injury patients in Japan: a long term review. Paraplegia 1988; 26: 165-176.

7 De Vivo MJ, Richards JS. Community reintegration and quality of life following spinal cord injury. Paraplegia 1992; 30: $108-112$.

8 Van Asbeck FWA, Raasden H, Van De Loo ML. Social implications for persons 5-10 years after spinal cord injury. Paraplegia 1994; 32: 330-335.

9 Berard EJJ et al. Etude des besoins des blessés médullaires vivant au domicile. In: Renconotres Autour du Blessé Médullaire. Masson: Paris, 1990, pp 202-207.

10 Daverat $\mathrm{P}$ et al. Early prognostic factors for walking in spinal cord injuries. Paraplegia 1988; 26: 255-261.

11 Chehrazi B, Wagner F, Collins W, Freedman D. A scale for evaluation of spinal cord injury. J Neurosurg 1981; 54: 310-315.

12 Radloff LS. The CES-D scale: a self-report depression scale for research in the general population. Appl Psychol Meas 1977; 1: 385-401.

13 Hamilton BB et al. A uniform data system for medical rehabilitation. In: Furher MJ (ed) Rehabilitation Outcomes. Brooks: Baltimore, 1987, pp 137-147.

14 Wood-Dauphinee SL et al. Assessment of global function: The Reintegration to Normal Living Index. Arch Phys Med Rehabil 1988; 69: 583-590.

15 Fenollosa $\mathrm{P}$ et al. Chronic pain in the spinal cord injured: statistical approach and pharmacological treatment. Paraplegia 1993; 31: 722-729.

16 Menter RR et al. Impairment, disability, handicap and medical expenses of persons aging with spinal cord injury. Paraplegia 1991; 29: 613-619.

17 Einsenberg MG, Saltz CC. Quality of life among aging spinal cord injured persons: Long term rehabilitation outcomes. Paraplegia 1991; 29: 514-520.

18 Fuhrer MJ et al. Depressive symptomatology in persons with spinal cord injury who reside in the community. Arch Phys Med Rehabil 1993; 74: 255-260. 